\title{
A RESONANT CAVITY FOR SINGLE-SHOT EMITTANCE MEASUREMENT*
}

\author{
J. S. Kim ${ }^{+}$, FARTECH, Inc., San Diego, CA, 92122 \\ C. D. Nantista, D. H. Whittum, R. H.Miller, S. G.Tantawi, SLAC, CA 94309 \\ A. W.Weidemann, University of Tennessee, Knoxville, TN 37996
}

\begin{abstract}
We present a non-invasive, resonant cavity based approach to beam emittance measurement of a shot-toshot non-circular beam pulse of multi-bunches. In a resonant cavity, desired field components can be enhanced up to $Q_{L \lambda} / \pi$, where $Q_{L \lambda}$ is the loaded $Q$ of the resonance mode $\lambda$, when the cavity resonant mode matches with the beam operating frequency. In particular, a Quad-cavity, with its quadrupole mode at beam operating frequency, extracts the beam quad-moment exclusively, utilizing the symmetry of the cavity and some simple networks to suppress common modes. Six successive beam quadrupole moment measurements, performed at different betatron phases in a linear transport system, allow us to determine the beam emittance, i.e., the beam size and shape in the beam's phase space. One measurement alone provides the rmsbeam size if the beam position is given, for instance, by nearby beam-position-monitors. This paper describes the basic design and analysis of a Quad-cavity beam monitoring system.
\end{abstract}

\section{INTRODUCTION}

RMS beam emittance is a key beam parameter, along with beam position, for accelerator operations[1]. Currently single-pulse emittance measurement is not available, and thus beam tune-up time takes from hours to days. A pulse-to-pulse size measurement based on striplines has been proposed previously, by Miller, et al.[2]. We have extended the idea of a resonant cavity[3] as a beamline instrument for beam emittance measurement. Advantages over a stripline configuration include: (1) stronger beam and desired cavity mode coupling, i.e., larger $[R / Q]$; and (2) high signal-to-noise ratio by resonance of a cavity mode at the bunch frequency.

The resonant cavity approach for emittance measurement is to employ a resonant cavity monitor operated in the quadrupole mode. This provides a voltage phasor output proportional to the product of charge and the beam moment $\left\langle x_{L}^{2}-y_{L}^{2}\right\rangle$ in the linac coordinates, phased with the beam. A series of such monitors placed in a FODO lattice, and separated by adequate machine phase advance, permits one to deconvolve beam matching parameters, and rms emittance.

* Work supported by US Department of Energy under the grants DE-FG03-98ER82574, and DE-AC03-76SF00515.

+kimjs@far-tech.com
The Quad-cavity is designed, by symmetry, to have a quadrupole mode that is exclusively excited by the beam's quadrupole moment, and thus, for a flat beam, is indicative of its size. In order for maximum coupling between the beam quadrupole moment $\left\langle x_{L}^{2}-y_{L}^{2}\right\rangle$ and the cavity quad-mode, the cavity must be rotated by 45 degrees along the linac axis as in Figure 1.

Parasitic common modes, i.e. modes other than the quad-modes, are minimized by: (1) separating the other resonance mode away from the quad-mode by at least 1 $\mathrm{GHz}$; (2) maximizing the beam and quad-mode coupling by optimizing the cavity length along the beam pipe; (3) utilizing symmetry in simple network to cancel out parasitic modes, and (4) using for this network waveguide in which the lower modes are cutoff. The electric field can be reduced by many orders of magnitude at a frequency about $1 \mathrm{GHz}$ away from the mode frequency. Further, the quad mode is the lowest mode of odd-odd symmetry with respect to the planes of $\mathrm{x}=0$ and $\mathrm{y}=0$, in the cavity coordinates. Thus, the monopole and dipoles can be superimposed away via proper hybrid $\mathrm{T}$ connections, as shown in Figure 1.

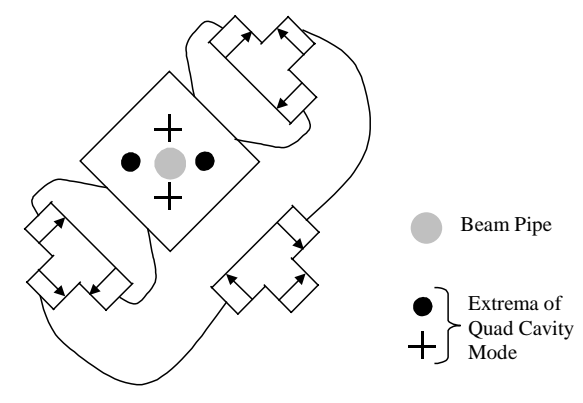

Figure 1. Cavity orientation in the beamline.

\section{CIRCUIT MODEL ANALYSIS}

The time-behavior of the voltage radiated from a beamdriven cavity mode may be described as a driven, damped, simple harmonic oscillator. With a drive term proportional to beam current $I_{b}$ and appropriate geometric factors and damping inversely proportional to the loaded quality factor of the mode $Q_{L \lambda}$,

$$
\left(\frac{d^{2}}{d t^{2}}+\frac{\omega_{\lambda}}{Q_{L \lambda}} \frac{d}{d t}+\omega_{\lambda}^{2}\right) V_{\lambda}\left(t, r_{\perp}\right) \approx-2 k_{\lambda} \frac{d}{d t} I_{b} X_{\lambda}\left(r_{\perp}\right),
$$

where we abbreviated $X_{\lambda}=1,\langle x\rangle,\langle x y\rangle$, and $k_{\lambda}=k_{m}, k_{d}^{\prime}, k_{q}^{\prime \prime}, k_{d}^{\prime} \equiv k_{d} / x$ for x-dipole, $k_{q}^{\prime \prime} \equiv k_{q} /\left(x^{2} y^{2}\right)$ for quad. We assumed a perfect match on the output line. The 
solution of the circuit equation for the induced mode voltage driven by a single-bunch with a Gaussian distribution in z of rms-length $\sigma_{t}$, may be expressed as a damped sinusoidal signal when

$$
\omega_{\lambda} \sigma_{t}<<1 \text {. Further, for } Q_{L \lambda}>>1 \text { : }
$$

$$
\lambda(t, t \underline{r})=2 \text { 质 } Q X_{\lambda} \Re\left[\exp \left(-\Gamma\left(t-t_{b}\right)\right)\right]
$$

where $\Gamma \equiv \frac{\omega_{\lambda}}{2 Q_{L \lambda}}-i \omega_{\lambda}$ and $\Re$ refers to the real part of the function. The amplitude of the sinusoidal voltage is proportional to $\left|V_{\lambda}\right|$. Multi-bunch responses can be obtained by summing over the single bunch responses at times delayed by the bunch interval $\tau$. For a train of $\mathrm{N}$ bunches, the amplitudes of the sinusoidal voltage of the $\ell$ th-bunch are,

$$
\left|V_{\lambda}^{N}\left(t_{\ell}\right) / V_{\lambda}^{N}\left(t_{0}\right)\right|=|(1-\exp (-\Gamma \ell \tau)) /(1-\exp (-\Gamma \tau))|
$$

where $t_{\ell}=t_{0}+(\ell-1) \tau$ for $\ell=1,2, \cdots, N$, and for $t>t_{N}$

$$
\left|\ell^{X}\left(\Delta t_{N}\right)\right|=\left|V^{N}\left(t_{N}\right) \exp \left(-\Gamma\left(t-t_{N}\right)\right)\right| .
$$

Figure 2 shows the amplitudes of voltages of 1000 bunches, filling every potential bucket, when the mode frequency is at perfect resonance with the bunch frequency (upper curve), at a frequencies mismatched by $\delta f=f_{0} /(2 Q)=7.6 \mathrm{MHz}$ with $Q=750$ (middle curve), and by $2 \delta f$ (bottom curve).

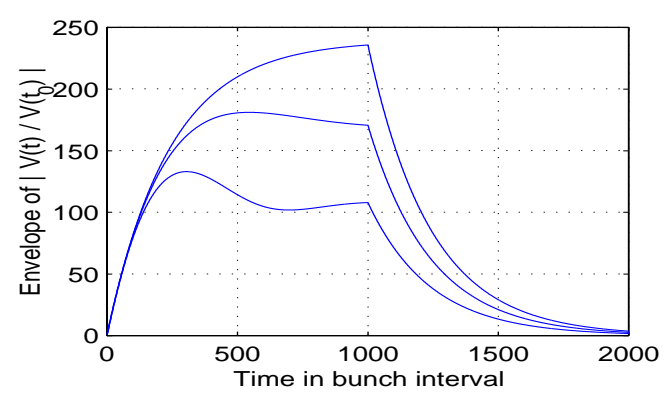

Figure 2. Amplitudes of cavity voltage at resonance (top), and off resonance by $\delta f=f_{d} /(2 Q)$ and by $2 \delta f$ (bottom).

The asymptotic voltage for infinitely many bunches is shown in Figure 3 for $0.9 \omega_{R F} \leq \omega_{\lambda} \leq 3.2 \omega_{R F}$, where $\omega_{R F}$ is the accelerator rf frequency at which the beam is bunched.

$$
\operatorname{Lim}_{N \rightarrow \infty}\left|V_{\lambda}^{N}(t)\right|=\left|\exp \left(\frac{\pi}{Q_{L \lambda}} \frac{\omega_{\lambda}}{\omega_{R F}}-2 \pi i \frac{\omega_{\lambda}}{\omega_{R F}}\right)-1\right|^{-1} .
$$

Approximating the asymptotic value around integer values as $\omega_{\lambda} / \omega_{R F}=n_{g}+\delta$, we obtain that the output voltage of the resonant mode is enhanced at multiples of the fundamental resonance by the factor of $Q_{L} /\left(\pi n_{g}\right)$, a large number but decreases with $n_{g}$, the bunch separation in terms of potential minima. The enhancement of the desired signal, suppression of the unwanted signals, and practical fabrication issues such as tolerance determine the design specification.

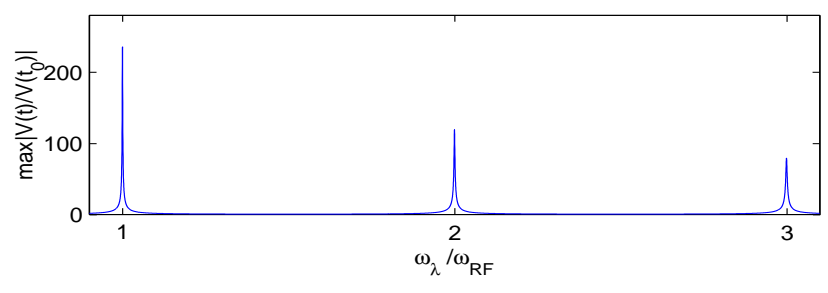

Figure 3. Asymptotic cavity voltage amplitudes.

\section{QUAD-CAVITY DESIGN}

Based on the basic resonance concept described in previous sections, we present a specific design of a Quadcavity for beam size monitoring or emittance measurement applicable for X-band accelerators operating at $11.424 \mathrm{GHz}$. First, we choose some design parameters specific to accelerators. The diameter of the beam pipe attached to the Quad-system is chosen as $1 \mathrm{~cm}$, large enough for an X-Band linac (with typical iris diameter of $8 \mathrm{~mm}$ ). Considering resonance enhancement effect and tight tolerance requirement in fabrication, with increasing $\mathrm{Q}$, we choose the external $\mathrm{Q}$ value around 750 , whose FWHM of the resonance mode energy is $15 \mathrm{MHz}$. With these chosen parameters, we arrived at an optimized quadcavity design, shown in Figure 4, that could permit a detection of an rms-beam size under 100 microns, after detailed numerical simulations using electromagnetic field-solvers [4][5]. The main design parameters are summarized in Table 1.

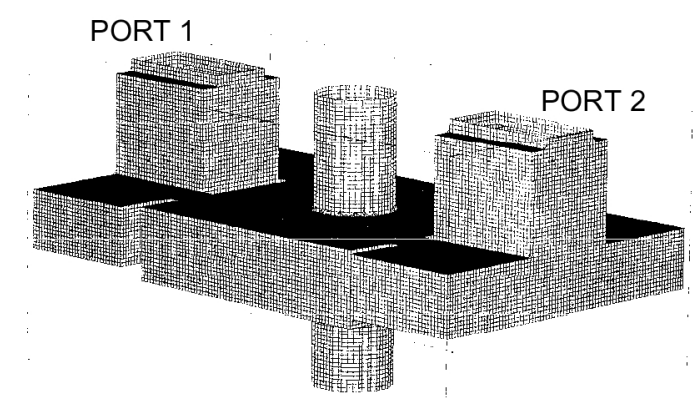

Figure 4. Quad-cavity and two hybrid tee geometry, with shortened waveguides, in mesh from the GdfidL code [4].

The pillbox resonance frequencies of the cavity in Table 1 are $5.8 \mathrm{GHz}$ (monopole), 9.1 and $9.3 \mathrm{GHz}$ (dipoles), 11.6 $\mathrm{GHz}$ (quad) and $12.84 \mathrm{GHz}$ (next higher mode). The perturbation presented by the four couplings irises, which brings the quad-mode to $11.424 \mathrm{GHz}$, should not significantly alter this mode spacing. 
